\title{
FREE-SURFACE WAVE DAMPING DUE TO VISCOSITY AND SURFACTANTS
}

\author{
J.-M. Vanden-Broeck ${ }^{(1)}$ and B. Spivak ${ }^{(2)}$ \\ (1) School of Mathematics, University of East Anglia, Norwich NR4 7TJ, UK \\ (2) School of Engineering, University of Tel Aviv, 69978, Israel
}

\section{Introduction}

The steady wave pattern developed on the free surface in inviscid two-dimensional fluid domain is well known. In this work, we investigate the influence of a thin film of insoluble material on air-liquid interface, on the wave characteristics. The linearized version of the titled problem in a two-fluid medium has been considered by Miloh et al [1]. We employ a quasi-potential approximation and assume that the main flow is potential, while the effect of surfactants enters the problem via Bernoulli equation. Thus, the problem is formulated in terms of a single potential function. The wave amplitude, consistent with the approximation made may be moderate. We consider a pressure distribution moving to the left with constant velocity $c>0$, on a two-dimensional deep water air-liquid interface, on which there exists a monolayer film of surface active agents. The equations and boundary conditions are expressed in terms of orthogonal curvilinear coordinates $(s, n)$. Here $s$ denotes arclength along the free surface and $n$ denotes distance along the straight line perpendicular to the interface, considered positive in the outwards direction (i.e. from the liquid to the air). The pressure problem is formulated as a system of nonlinear integrodifferential equations for the unknowns on the free surface. These equations are discretized and converted into a system of nonlinear algebraic equations which are solved by Newton's iterations.

\section{Governing equations and numerical procedure}

We consider a two-dimensional distribution of pressure $P$ (with compact support) moving to the left at a constant velocity $c$ at the surface of a fluid of infinite depth and constant density $\rho$. This can be interpreted as an inverse problem for a "two-dimensional" contour moving at a constant velocity $c$ at the surface of a fluid. Here the shape of the contour is not known a priori but is given at the end of the calculations by the shape of the free surface below the support of the pressure distribution. We seek steady solutions in a frame moving with the pressure distribution. We choose Cartesian coordinates with the $y$-axis directed vertically upwards. We introduce dimensionless variables by choosing $c$ as the unit velocity and $c^{2} / g$ as the unit length. Here $g$ is the acceleration of gravity.

The full boundary conditions for a Newtonian type interface in the presence of surfactants are given in Edwards et al [2]. The derivation of these boundary conditions is based on the so called Boussinesq-Scriven constitutive relationship (Scriven [3]) which is expressed in terms of two quantities $\mu^{s}$ and $\kappa^{s}$, the interfacial and dilatational viscosities respectively. We combine these boundary conditions with the quasi-potential approximation developed by Ruvinsky et al [4]. This approximation is valid for slightly viscous fluids. The velocity field is separated into a potential part and an irrotational one. The 
resulting coupled equations enable one to solve the potential part and deduce from it the (assumed small) irrotational one. The resulting boundary condition on the free surface for the potential function $\phi$ is

$$
\frac{1}{2}\left(\frac{\partial \phi}{\partial s}\right)^{2}+y+P-\frac{2}{R_{e}} \frac{\partial^{2} \phi}{\partial s^{2}}\left(1+K B_{0}\right)-K\left(W_{e}-M_{r} \frac{\partial \phi}{\partial s}\right)=\frac{1}{2}
$$

Here

$$
R_{e}=\frac{c^{3}}{g \nu}
$$

is the Reynolds number,

$$
B_{0}=g \frac{\kappa_{s}+\mu_{s}}{2 \rho \nu c^{2}}
$$

the Boussinesq number,

$$
W_{e}=g \frac{\sigma_{0}}{\rho c^{4}}
$$

the Weber number and

$$
M_{r}=g E_{0} / \rho c^{4}
$$

the Marangoni number. Here $\nu$ is the kinematic viscosity, $E_{0}$ the Gibbs elasticity, $K$ the curvature of the free surface and

$$
\sigma=\sigma_{0}-\frac{E_{0}}{c} \frac{\partial^{2} \phi}{\partial s^{2}}
$$

the variable surface tension.

Next we introduce, in addition to the potential function $\phi$, the stream function $\psi$. Without loss of generality, we chose $\psi=0$ on the free surface. Following Asavanant and Vanden-Broeck [5], it can be shown that

$$
x^{\prime}(\phi)-1=-\frac{1}{\pi} \int_{-\infty}^{\infty} \frac{y^{\prime}(\varphi)}{\varphi-\phi} d \varphi
$$

Here we use $\phi$ and $\psi$ as independent variables and denote by $x(\phi)$ and $y(\phi)$ the values of $x$ and $y$ on the free surface $\psi=0$. The prime denotes derivative with respect to $\phi$.

We then express all the derivatives in (1) in terms of $x(\phi)$ and $y(\phi)$. This can easily be done by noting that

$$
\begin{gathered}
\frac{\partial \phi}{\partial s}=\left(x^{2}+y^{\prime 2}\right)^{-1 / 2} \\
\frac{\partial^{2} \phi}{\partial s^{2}}=-\frac{x^{\prime} x^{\prime \prime}+y^{\prime} y^{\prime \prime}}{\left(x^{\prime 2}+y^{\prime 2}\right)^{2}} \\
K=\frac{x^{\prime} y^{\prime \prime}-y^{\prime} x^{\prime \prime}}{x^{\prime 2}+y^{\prime 2}}
\end{gathered}
$$


For given values of $R_{e}, B_{0}, W_{e}$ and $M_{r}$, relations (1), (7)-(10) define a system of integrodifferential equations for the two unknown functions $x(\phi)$ and $y(\phi)$ This system is discretized and the resulting algebraic equations are solved by Newton's iterations. In our calculation we chose

$$
P=\epsilon \exp \frac{b}{\phi^{2}-a^{2}} \quad \text { for } \quad|\phi|<a
$$

and $P=0$ for $|\phi| \geq a$.

Once $x(\phi)$ and $y(\phi)$ are known, the free-surface vorticity $\omega_{s}$ is determined from

$$
\omega_{s}=2 K+2 B_{0} \frac{\partial^{3} \phi}{\partial s^{3}}-R_{e} M_{r} \frac{\partial^{2} \phi}{\partial s^{2}}
$$

In (12), $K$ and $\frac{\partial^{2} \phi}{\partial s^{2}}$ are given by (10) and (9) and $\frac{\partial^{3} \phi}{\partial s^{3}}$ is calculated by

$$
\frac{\partial^{3} \phi}{\partial s^{3}}=-\left[\frac{x^{\prime} x^{\prime \prime}+y^{\prime} y^{\prime \prime}}{\left(x^{\prime 2}+y^{\prime 2}\right)^{2}}\right]^{\prime}\left(x^{2}+y^{2}\right)^{-1 / 2}
$$

The numerical results show that there are in general two trains of waves on the free surface (a train of short waves at the front of the distribution of pressure and a train of longer waves at the back). Both trains are damped by viscosity. We will discuss how the various parameters $R_{e}, B_{0}, W_{e}$ and $M_{r}$ affect the rate of damping.

\section{Acknowledgments}

The authors thank T. Miloh and G. Zilman for drawing their attention to this problem and for constructive discussions.

\section{References}

[1] T. Miloh, O. Oshri and G. Zilman (1998). Free surface flow with viscosity (ed P.A. Tyvand), Computational Mechanics Publication, Southhampton, 117.

[2] D.A. Edwards, H. Brenner and D.T. Wasan (1991). Interfacial Transport Processes and Rheology, Butterworth-Heineman, Boston.

[3] L.E. Scriven (1960). Chem. Eng. Sci. 12, 98.

[4] K.D. Ruvinsky, F.I. Feldstein and G.I. Freidman (1991). J. Fluid Mech. 230, 339.

[5] J. Asavanant and J.-M. Vanden-Broeck (1994). J. Fluid Mech. 273, 109. 